\title{
Remote power strip with zero standby power controllable from every direction
}

\author{
Daichi Koide ${ }^{\mathrm{a},{ }^{*}, \text { Kazunari Yoshikawa }}{ }^{\mathrm{a}}$, \\ Akira Yamawaki ${ }^{\mathrm{a}, *}$ \\ ${ }^{a}$ Kyushu Institute of Technology, 1-1 Sensui-cho, Tobata-ku, Kitakyushu-shi, Fukuoka, 804-8550, Japan \\ *yama@ecs.kyutech.ac.jp
}

\begin{abstract}
The standby power consumption of the home appliances occupies $5-10 \%$ of the total annual power consumption in Japan. Thus, it is important to eliminate the standby power for the earth environment. However, turning off the standby power consumption of the home appliances is very bothersome job because the power plug of each appliance must be disconnected from the power strip. So we have developed a remote control power strip that standby power is zero by infrared remote control to eliminate that bothersome job. Since the proposed power strip contains the light receiving part, the installation location is strictly limited to the space where the user can irradiate the power strip from the remote controller. This paper attempts to separate the light-receiving part from the proposed power strip. As a result, the proposed power strip can be installed to any location. To allow the user to irradiate the light receiving part from every direction, we introduce a hemispherical mirror that reflect the light from any direction to the receiving part. The preliminary experiment shows that the switching operation can be accomplished actually in all directions.
\end{abstract}

Keywords: standby power requirement, half-spherical mirrors, Power LED.

\section{Introduction}

The standby power consumption of the home appliances occupies $5-10 \%$ of the total annual power consumption in Japan. It is equivalent to approximately 3.6 of the nuclear power plants ${ }^{(2)}$. The reduction of standby power is very important for the earth environment.

To eliminate the standby power, we must disconnect the plug from the power strip or switch off the main switch of the electronic product. However, this job is very bothersome. For example, we must approach each electronic product, look for the power strip behind the furniture and find the correct plug.

To avoid such bothersome job automatically, there is the proposal which employs a super condenser attached to an electronic product ${ }^{(3)}$. This technology previously charges the electric power to the condenser while the product is running. When the product moves to the standby mode, the electric power previously charged in the condenser is used instead of the commercial AC supply. However, this only borrows the power in advance which will be consumed in the standby mode. That is, the standby power is not eliminated.

We have developed a remote control power strip that standby power is zero by infrared remote control ${ }^{(1)}$. We have verified switching operation. We did a calculation of power consumption and battery life.

Since the proposed power strip contains the light receiving part, the installation location is strictly limited to the space where the user can irradiate the power strip from the remote controller. This paper attempts to separate the light-receiving part from the proposed power strip. As a result, the proposed power strip can be installed to any location. To allow the user to irradiate the light receiving part from every direction, we introduce a hemispherical mirror that reflect the light from any direction to the receiving part. Finally, we confirm whether the switching operation is performed actually in all directions. 


\section{Remote controlled power strip}

\subsection{System overview}

Fig. 1 shows a diagram of the overall system. When the remote controller irradiates a strong infrared light to the light receiving part, the electromotive force is generated from the photodiode. The latching relay connects or disconnects the AC line by utilizing the electromotive force. When AC line is disconnected, the standby power of the electronic devices connected to the power strip becomes zero.

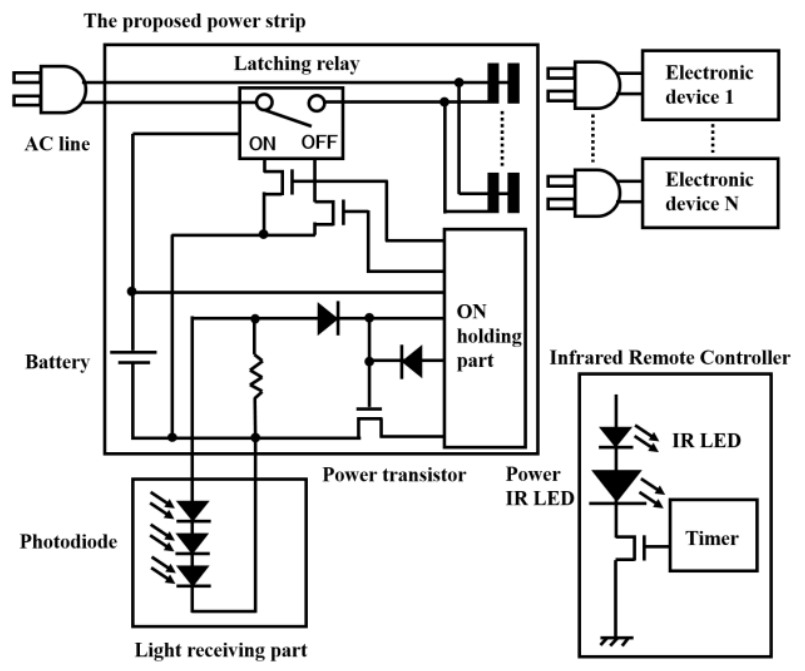

Fig. 1. System summary.

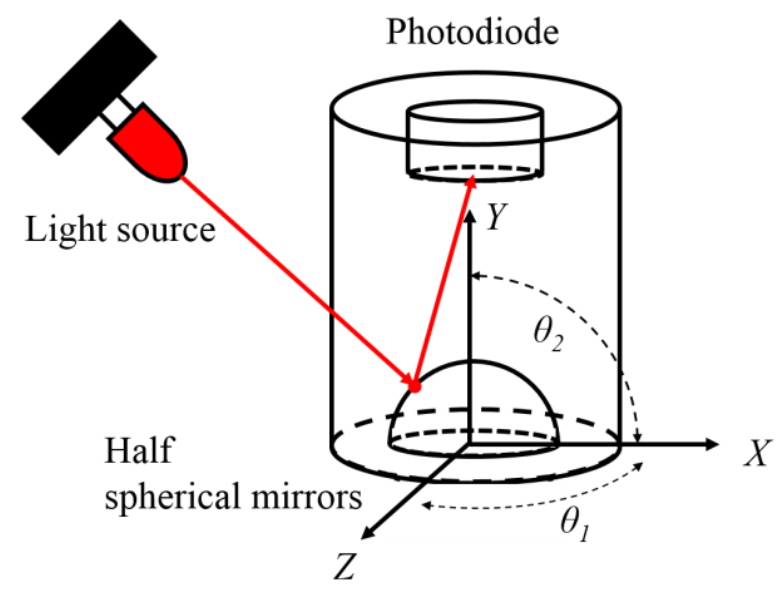

Fig. 2. Light receiving part.

\subsection{Light receiving part}

Fig. 2 shows a diagram of the Light receiving part. A cylindrical transparent box has a half spherical mirrors. On top of the half spherical mirrors there is a photodiode. The $\theta 1$ is the angle of the $X$ and $Z$. The $\theta 2$ is the angle of the $X$ and Y. Half Spherical mirrors can be display the range of $\theta 1=360\left[^{\circ}\right], \theta 2=180\left[^{\circ}\right]$.If the light source is higher than the half spherical mirrors. The light reflected by the half spherical mirrors photodiode is received.

\subsection{Power Strip}

Fig.3 (a) shows a detailed block diagram of the proposed remote controlled power strip. Photodiode generates an electromotive force when irradiated strong infrared. Power transistor performs the connection and disconnection of GND line. Latching relay 1 performs the connection and disconnection of the AC line. Latching relay 2 records the state of the AC line indicating whether it is disconnected or connected. A microcomputer manages the relays.

\subsection{Remote Controller}

Fig. 3 (b) shows a block diagram of the infrared remote controller. The infrared remote controller uses an infrared LED for transmitting infrared normal operation code. The power LED that emits a strong infrared is used to turn on and turn off the proposed power strip.

\subsection{Description of Operation}

Latching relay 1 performs connection or disconnection of the AC line. Latching relay 2 is used to record the on-off state of the latching relay 1 . When latching relay 2 is turn off, outputs ' 0 ' in the conduction state. When latching relay 1 is turn off, the electronic device is a standby state. When the latching relay 2 is turn on, outputs ' 1 ' of the insulating state. When latching relay 1 is turn on, the electronic equipment is operational.

Remote Controller is for turning on and off the power to the remote control receiver device. When the button of the power-on is pressed, a light pulse is emitted from the power LED. When the button of the power-off is pressed, a light pulse is emitted from the power LED. This is also the same as when the power is turned on. When other buttons to control the electronic product are pushed, the infrared light modulated by a pulse of $38[\mathrm{kHz}]$ is irradiated from the infrared LED. This is the same frequency as the normal remote control. 
AC line is the state of off $\rightarrow$ on and on $\rightarrow$ off. We describe AC line turn of off $\rightarrow$ on. During standby, the power-on transistor and latching relay 1 is turned off. Since the GND is disconnected, the microcomputer does not require standby power. In addition, since the AC line is disconnected, an electronic product connected to the power strip does not require standby power.

When receiving a strong infrared pulse indicating power on from the remote controller, the photodiode generates an electromotive force. Whereby the power transistor is on.

Then, the microcomputer continues to turn on the power transistor. The output of the latching relay 2 is ' 0 '. This means that the $\mathrm{AC}$ line is disconnected by the latching relay 1.The microcomputer turns on the latching relay 1 . As a result, the electronic product connected to the power strip goes to the normal operation. To record that the electronic device is a normal operating state, the microcontroller turns off the latching relay 2 and make its output 1 . Microcomputer is turned on the power transistor. The microcomputer makes some delay to ensure that the latching relay 1 and 2 stay at the stable state. Then, the microcomputer turns off the power transistor. AC line is turned on. Electronic devices operates as usual. When the power transistor is turned off, the microcomputer is a non-powered state.

Next, let us explain the operations turning off the power strip. During an electronic product connected to the power strip is running, the power-on transistor is turned off. Since the GND is disconnected, the microcomputer, the proposed power strip, does not require any standby power. When receiving a strong infrared pulse from the remote control, the photodiode generates an electromotive force. Whereby the power transistor is on. Then, the microcomputer continues to turn on the power transistor. Since the output of the latching relay 2 is ' 1 ', the microcomputer recognizes that the electronic equipment is in the normal operation. The microcomputer turns off the latching relay 1 to turn off the electronic product. To record that the electronic equipment is again in standby state, the microcomputer turns on latching relay 2 . As a result the output of the latching relay 2 becomes 0 .The microcomputer waits for the latching relay 1 and 2 going to the stable state. Then, the microcomputer stops to continue to turn on the power transistor. As a result, the power transistor and latching relay 1 are both turned off. That is, the whole system does not consume any power.

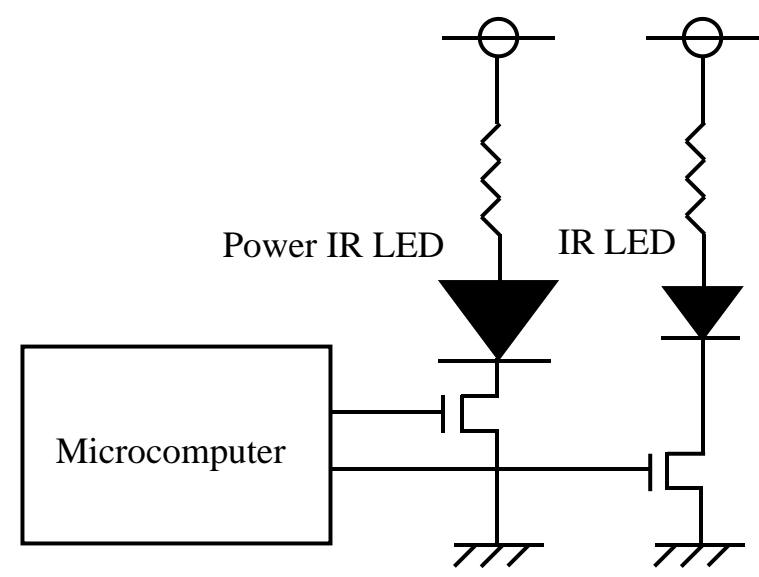

(a) Remote Controller.

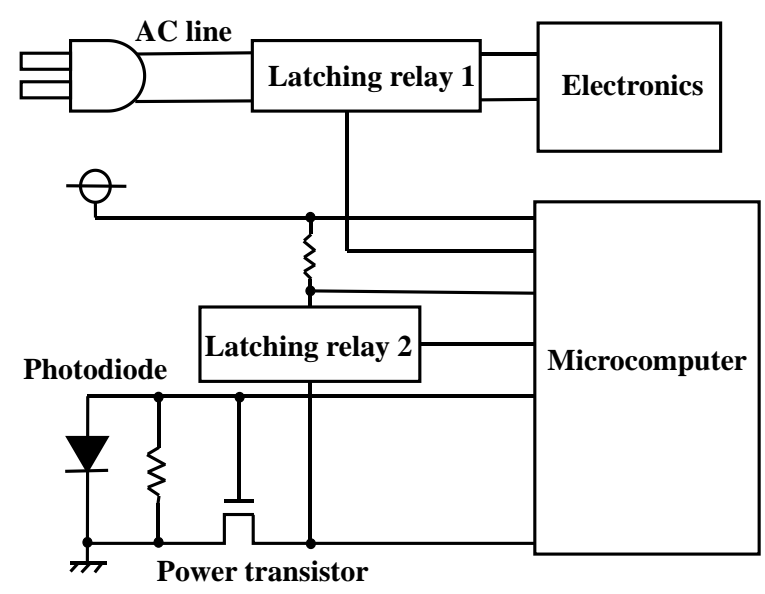

(b) Remote Power Switch.

Fig. 3. Circuit diagram transceiver.

\section{Operation verification}

\subsection{Experimental Setup}

In this verification, the distance between the light receiving part and the power strip was $2[\mathrm{~m}]$. We used the infrared light emitter developed instead of the remote controller. Transmission pulse width of the infrared light emitter is 18 [ms]. The diameter of the half spherical mirrors was $5[\mathrm{~cm}]$. The distance between the Half Spherical mirrors receiving element is a $2[\mathrm{~cm}]$.

\subsection{Distance Effect from Light Emitter to Receiver}

We measure the electromotive force when changing the distance between the light-receiving part and the infrared light emitter. We set at an angle between the light-receiving part and the infrared light emitter of the $\theta 1=\theta 2=0\left[^{\circ}\right]$. We changed by $5[\mathrm{~cm}]$ the range from $0 \sim 35[\mathrm{~cm}]$. 


\subsection{Switching from any direction}

We set the distance $15[\mathrm{~cm}]$ from the infrared emitter to the light receiving part. We have varied a range of $\theta 1=0 \sim 360\left[^{\circ}\right]$ of the half spherical mirror by $45\left[^{\circ}\right]$. We have changed by $15\left[^{\circ}\right]$ for the range of $\theta 2=0 \sim 90\left[^{\circ}\right]$. Every angle, we have recorded the electromotive force of the photodiode at the time of switching operation.

\subsection{Validation result}

Fig. 4 shows the verification result of distance effect from light emitter to receiver. We were able to confirm the on-off of the AC line of the distance between the lightreceiving part and the infrared light emitter to $25[\mathrm{~cm}]$. We have found that the AC line cannot be turned on-off in the threshold voltage of the power transistor $2[\mathrm{~V}]$.

Fig. 5 shows the verification result of switching from any direction. Fig. 5 (a) is the electromotive force when changing the $\theta 1$. Fig. 5 (b) is the electromotive force when changing the $\theta 2$. We have confirmed of the AC line on-off at all angles. We found that can be received from any direction light receiving part. Electromotive force at all angles was higher than $2[\mathrm{~V}]$ threshold voltage.

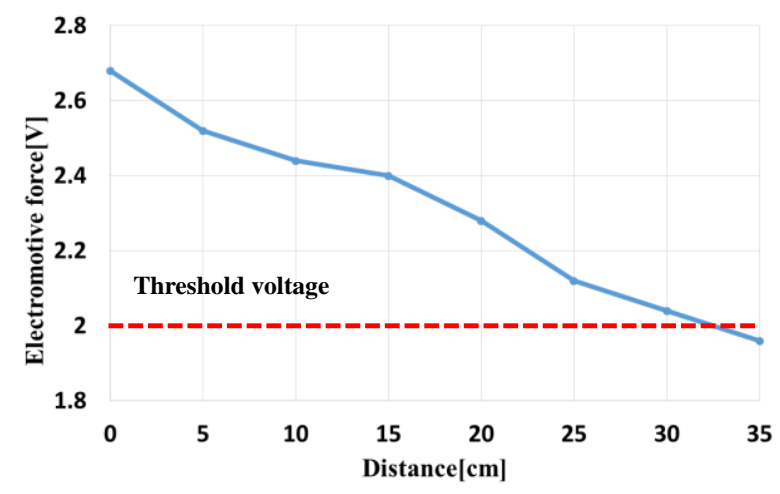

Fig. 4. Electromotive force.

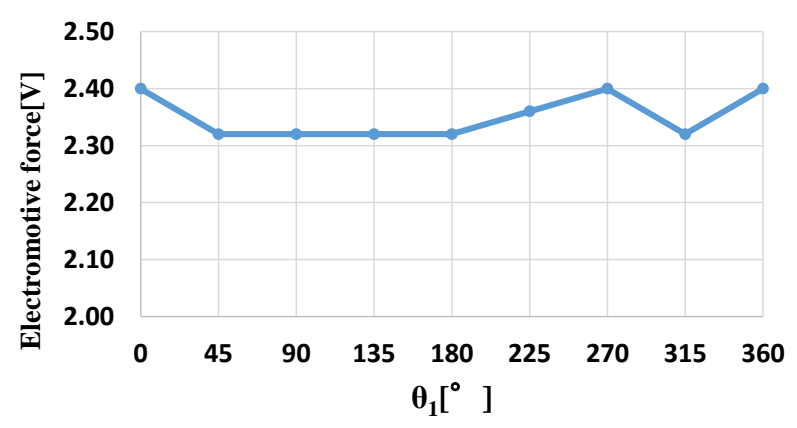

(a) Horizontal direction.

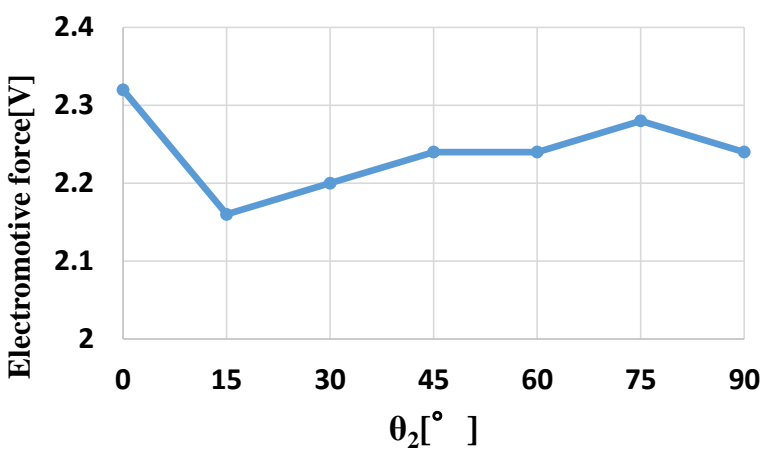

(b) Vertical direction.

Fig. 5. Electromotive force.

\section{Conclusions}

We used a half spherical mirrors. We developed a remote power strip of standby power zero by an infrared remote control. We confirmed the on-off of the AC line although the infrared light from the remote controller is reflected by the half spherical mirror. We have found that the AC line is not turned on-off in the threshold voltage of the power transistor $2 \mathrm{~V}$ when distance between the lightreceiving part and the infrared light emitter is far. It has become possible to install only the light receiving part in a location that is easily illuminated by releasing the proposed strip and the light receiving part. We were able to widen considerably the range of light receiving $\left(\theta 1=0 \sim 360\left[^{\circ}\right]\right.$, $\left.\theta 2=0 \sim 90\left[{ }^{\circ}\right]\right)$. Future, the remote control transmitter part to be able to store the signal of equipment. We will extend the communication distance of the proposed strip. We want to be able to operate the electronic equipment and power strip by a remote control.

\section{References}

(1) Daichi Koide, Mayu Yamanaka, Daisuke Tanaka,Takuya Itahashi, and Akira Yamawaki : "Remote controlled power strip to make standby power consumption zero", ICIAE2015, 2015

(2) National Institute of Advanced Nuclear Energy Safety Organization Planning Department : "2013 edition nuclear facility operation management annual report", Nuclear facility operation management annual report, Vol. 1, No. 1, pp. 1-526, 2013

(3) Hijikata KeiJiro : "Standby power zero watts of television by eco chip TM", ITE Journal, Vol. 1, No. 1, pp. 729-731, 2012 